\title{
Report for the Mental Health Commission on Admission of Young people to Adult Mental Health Wards in the Republic of Ireland
}

\author{
Dr. Sally E. Bonnar
}

December 2010 


\section{Introduction}

This report was commissioned by the Mental Health Commission to

1) Examine the factors contributing to the admission of children under 16 in 2010 to Adult Psychiatric Units and Paediatric wards

2) To examine the challenges for providers in meeting the mental health needs of the under 18s in the context of the addendum to the Mental Health Commission Code of Practice for the admission of children

3) To present a report of the findings and recommendation to the Mental Health Commission.

The report was based on documents pertaining to both Mental Health legislation in Ireland and to service strategic planning and delivery, a list of which can be found in Appendix 1. In addition the author visited Ireland on $16^{\text {th }}-20^{\text {th }}$ September and met with a number of individuals responsible for service delivery including a visit on site to services in Limerick where the Commission was particularly concerned about admission figures for young people to a local adult psychiatric ward. Further site visits were not conducted although the author did interview staff with national responsibilities both formal and informal in order to gain an Ireland wide perspective.

Although the report aims to be as comprehensive as possible, there may well be other views within the mental health community that differ from those already expressed.

The author has also used experience and strategic decision making from the Scottish context to inform the recommendations. Scotland has similar challenges in relation to geography and rurality to Ireland and can provide a useful comparator.

The recommendations are the author's own and intended to be a basis for discussion rather than a prescription. 


\section{Context}

2.1 The Republic of Ireland has a Mental Health Act enacted in 2001 that makes quite clear the process for detaining a child (under18 years) in hospital ( Section $25 \mathrm{ff}$ ). In addition, the Code of Practice in Relation to the Admission of Children under the Mental Health Act (2006) and its subsequent Addendum (2009) seek to clarify best practice in relation to the care and treatment of children and young people with mental health needs. These documents all make clear that over-riding principle that the best interests of the child are paramount. This is in keeping with similar guidance in other countries who subscribe to the United Nations' Convention on the Rights of the Child (1999). This is an important principle and it is clarified within the Mental Health Commission's own documentation that this may in exceptional circumstances lead to a young person under the age of 18 being admitted to and approved centre for adult patients. It is however right and proper that these occasions should be minimised as far as possible and that the Commission have oversight of when they occur in order to fulfill its role of protecting vulnerable patients.

2.2 Like many nations, provision of Child and Adolescent Mental Health Service (henceforth called CAMHS) has lagged behind provision for adults and in many cases has developed serendipitously with little in the way of strategic planning until recently. More recently in Ireland the "Vision for Change " policy document (2006) has included CAMHS in its planning for the development of Mental Health services and sets out recommendations for numbers of inpatient beds as well as supporting community teams. Details of numbers are laid out in Appendix II. Whilst there was a general welcome for this document, in that a focus was placed on strategic development of CAMH Services, there appear to be anxieties about its implementation. Of particular relevance to this report are the references in subsequent documents, including those from the Mental Health Commission, citing delays in implementation and continuing under-provision of Child and Adolescent inpatient beds. The recommendation in Vision for Change is 
for 108 inpatient beds to include 10 specifically dedicated to Secure Psychiatric care and 6/8 devoted to eating disorders. The report does not specify whether the remaining beds should be split between children and adolescents or what the proportion of such a split ought to be. At the present time there are 52 beds for children in Ireland assuming that the 2 new units in Galway and Cork can be opened in the autumn of 2010 as planned and fully commissioned. Discussion with staff in Limerick in particular suggested that there may be problems with commissioning all the beds due to recruitment and training issues. This is not an uncommon problem in CAMHS where there is already a low capacity base in community services and a limited pool from which to draw staff. A similar problem prevented the full commissioning of a new inpatient facility in Glasgow where only 20 of the planned 24 beds have been opened 18 months after commissioning.

2.3 Ireland is a predominantly rural country with discreet centres of population distributed mainly around the coastal areas. The HSE provides a National service through 4 operational units, 2 of which provide for the greater Dublin area. With a relatively small population, it is inevitable that some highly specialized services will have to be provided in a centralized manner. This leads to patients often having to travel long distances for treatment. Whilst this may be acceptable for acute episodes of treatment with back up being provided more locally, young people with mental illness often require prolonged periods of inpatient treatment that may last several months. Longer admissions and delayed discharges are associated with poorer provision of community services and especially with poor intensive support (Tier 4) services in local areas. It therefore becomes difficult to meet the needs of acutely mentally ill young people in units where they are both close to their own homes and have sufficiently skilled and trained staff to meet their needs. This is the well known problem of high need low volume services and is similar to the problems in Scotland where, if anything, the rurality problems are even more challenging. 


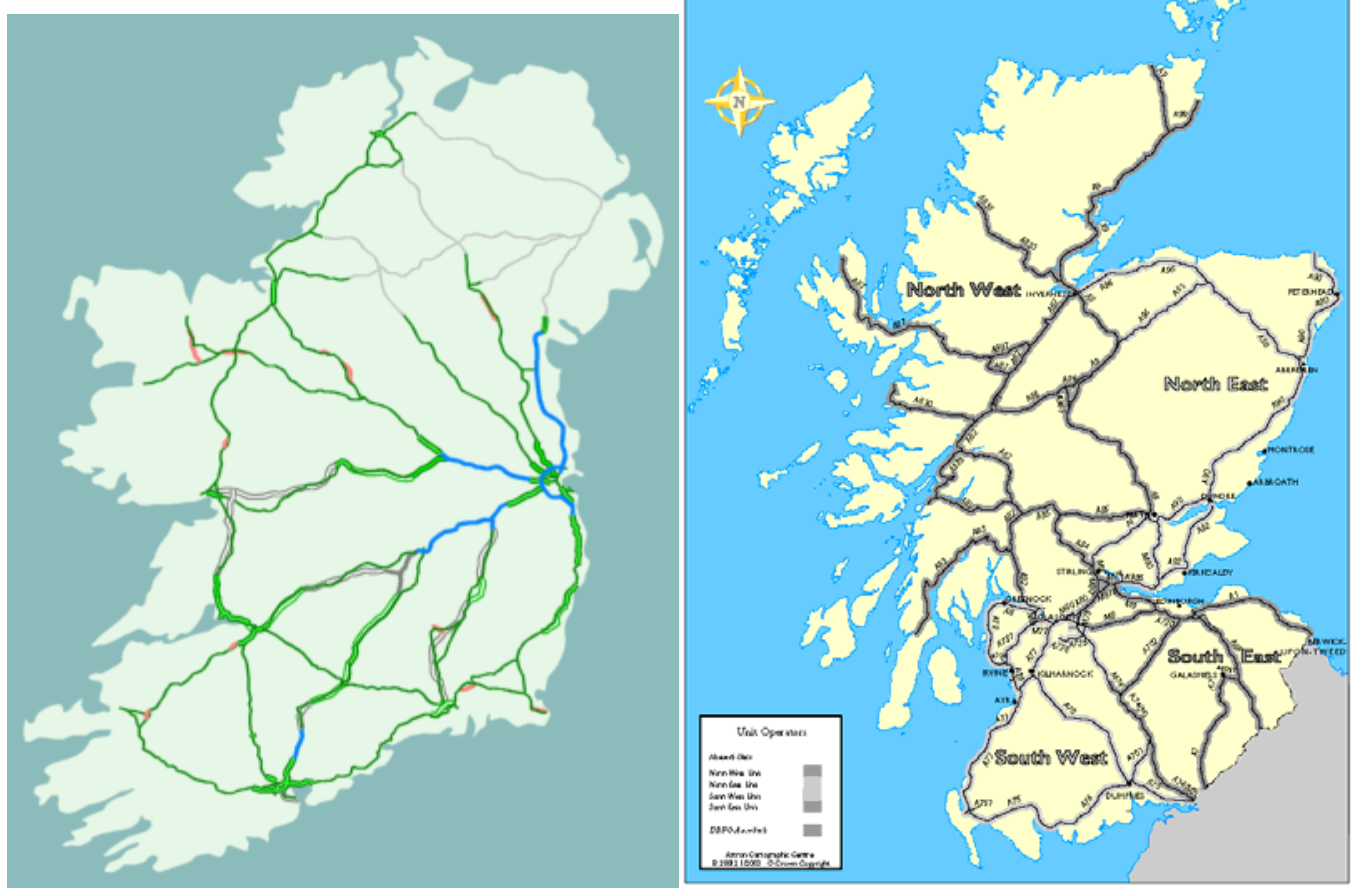

2.4 It is generally accepted that although Vision for Change has made clear recommendations for numbers and configurations of community teams for CAMHS, these have not yet been fully implemented and current capacity falls far short of what would be required to provide comprehensive community services across the country, including community Tier 4 services. Low capacity also inhibits the development of specialization within CAMHS. There is therefore little in the way of services for e.g. young people with drug and alcohol problems. This leads at times to inappropriate short term admissions that are not seen as the role of modern Child and Adolescent inpatient services and that in many other areas would be considered "social admissions", more appropriately dealt with by social services.

There is a challenge from transport infrastructure that means that whilst connections between Dublin and other population centres are good, transport across the county is more difficult. This makes parents (and to some extent clinicians) reluctant to admit their young people to units distant from their home because of time and financial constraints. Thus young people 
are on occasion preferentially placed in adult approved centres rather than face the lengthy journeys to specialist units.

2.5 Provision of other services, particularly Social Work service, for young people and their families is patchy and there is no out of hours provision social work provision other than in Dublin. at all for children and young people. Consequently much of the work that in other jurisdictions would be undertaken by social services inevitably falls to CAMHS. This is particularly acute where CAMH Services do provide out of hours cover, as is the case in Limerick. Cases presenting out of hours are most often crisis social care emergencies needing a short term solution. These cases are often high risk and highly anxiety provoking and it is difficult to resist the pressure to admit. However in many other places where out of hours social work exists they would not be admitted to a psychiatric facility. Because of the shortage of beds and distances involved in admitting to specialist inpatient units for young people, most of these emergency and out of hours cases end up having short term admissions to adult or paediatric wards.

\section{Findings}

It is important here to thank all those who contributed so willingly to the findings below and to acknowledge that all those whom I met were trying very hard to provide the best possible service to children and young people sometimes in very difficult situations.

3.1 Since the implementation of the Addendum to the Code of Practice for Admission of Children, the Mental Health Commission has collected figures to show that in the first six months, there was only 1 young person under the age of 16 admitted to an adult facility. However in the second 6 months, there were 9 admissions, 8 to one adult ward in Limerick. (See Appendix 3 for figures) In addition, it has become clear that in Limerick, two beds in a paediatric ward are being designated by the service for the use of the CAMH Service with CAMHS Consultants taking responsibility for those patients admitted. Although the majority of young people admitted to adult facilities are over 16, there is still a significant proportion 
under 16, and worryingly, one child as young as 13 . There is anxiety that with the approach of the next deadline for admission of under $17 \mathrm{~s}$, it will not be possible to accommodate these patients in appropriate Child and Adolescent facilities.

3.2 As previously noted, the Mental Health Act and subsequent codes of practice nowhere say that a young person should never be admitted to an adult facility. Indeed examples were cited where admission to an adult ward would be the most appropriate placement for some 16-17 year olds. Such examples included, young people with early onset adult type illness such as psychosis or bipolar disorder who were approaching their $18^{\text {th }}$ birthday and some young people who were in employment and would see themselves as adult. On a case by case basis it was felt that these and other such patients should have a degree of choice in where they were looked after.

3.3 The role of parents in choice of admission unit is a difficult one. It is clear that in Ireland, parental rights up to a young person's $18^{\text {th }}$ birthday are enshrined in constitutional law. There have been examples of courts ruling to admit young people to adult facilities because this was the parent's preference rather than to what might be considered a more appropriate adolescent unit. It is difficult to see how this can be changed other than by good argument. This would mean that even although the professionals and the Mental Health Commission may wish to prevent the inappropriate admission of young people, they may still be confounded by court rulings in favour of parents. Independent advocacy as enshrined in the Mental Health (Scotland) Act 2003 allows for the voice of young people to be heard apart from their parents. No such provision exists in Ireland although the Mental Health Commission has developed a very useful self-advocacy toolkit to enable young people to advocate for themselves.

3.4 There is a perceived disconnect between the admitting units for young people and the community teams from whom they receive their referrals. There are seldom beds available to accept admissions in an emergency and even planned 
admissions may take several weeks. Community teams can feel distant from the care of their young people making discharge planning difficult. Most community teams will make multiple referrals to different units in a search for a bed leading to duplication of effort and confusion for the inpatient units about who actually should be taking responsibility for these referrals.

3.5 Attitudes within the psychiatric community vary as to who is best place to look after young people with acute mental illness, particularly those in the 16-17 year old age bracket. Until recently these patients have been the clear responsibility of adult mental health services that see themselves as having the expertise in dealing with the types of illnesses that commonly present for the first time in this age group. It is clear that the principle of CAMHS looking after all patients under 18 is generally accepted but there is an acknowledgement that the services are not yet there to make this a reality. Some adult services would appear to be more willing than others to come to local arrangements for the care of the 16-17 year olds. Local arrangements seem to be key to the success of care packages for inpatients who are under 18 particularly in emergency situations.

3.6 As previously alluded to, there is a lack of capacity in community CAMH Services to provide comprehensive community treatment and care. Although there has been significant investment in staff and new teams have been set up, recruitment has not been easy and the numbers working in CAMHS still do not come near to those recommended by Vision for Change. In particular there has been little development of intensive community treatment teams that might assist with prevention of admission to inpatient care and facilitate early discharge thus making better use of the scarce inpatient resource. Generally outpatient teams are bogged down with responding to urgent situations and long waiting lists. Figures for recommended versus actual CAMHS capacity are presented in Appendix IV 
3.7 In Limerick there are a number of anomalies that make it more likely that their figures will be different from other services. Firstly there is a long history of CAMH services providing out of hours care and an emergency in-hours service is also provided. This has accustomed local health and social services to getting a rapid response from CAMHS. They are therefore at increased likelihood of being asked to deal with urgent situations. The service has worked out a transparent arrangement both with their adult colleagues and with paediatrics to provide urgent and planned care for their patients and have well constructed protocols for the care of these patients. This is based on their long term experience of restricted access to the Adolescent Unit in Galway especially in an emergency situation due to lack of a vacant bed. In relation to the use of paediatric beds, it would appear from services across the country that it is common practice to admit to children's wards especially in an emergency situation such as an episode of self harm. The difference in Limerick is that the beds are designated for the use of psychiatry and patients are explicitly under the care of a child and adolescent psychiatrist during their admission. In other parts of the country the patients in paediatric wards are admitted under the care of paediatricians and are generally only admitted for a very short period, i.e. overnight, whilst awaiting assessment after episodes of self harm. From experience, it is very difficult to access numbers for these admissions as they are not routinely recorded by reason for admission. It would seem therefore that at least to some extent, Limerick has been caught out by its attempt to be more transparent.

3.8 There is a relatively new attempt to develop a network approach across the current inpatient units to look at common pathways and referral protocols etc. This would appear to be a very sensible way forward but again there is, as yet, no involvement of community services in this network. There is still lack of clarity as to whether or not the final complement of 108 beds will be provided, especially in the difficult financial situation in Ireland at present. It should be noted that all the inpatient units are members of QNIC (Quality Network for Inpatient CAMHS), the Royal College of Psychiatrists quality 
network for inpatient CAMHS. Although this is a network run by the UK College, overseas units are eligible to join and the Irish inpatient units are all members. This is an important governance tool for services.

3.9 It was difficult to ascertain what the user's voice was in this debate. Involvement of users and carers in planning would seem to be at an early stage. Experience from Scotland suggests that the user and carer voice is very influential with planners and politicians and can positively influence clinical decision making. Whilst it is clear that parents have a major say in how their young people are looked after, it is less clear how the wishes and opinions of children and young people themselves are heard.

3.10 There are very real fears about the impact of the current financial crisis in Ireland. There is a recognition of the reality and the need for reduction in spending within health services although some assurance that CAMHS will be relatively protected. There does however seem to be an acceptance of the fact that further investment in CAMHS will be hard to come by.

3.11 The institution of the CAMHS Advisory group is a relatively new initiative that brings together a multi-disciplinary team to advise the Health Executive on CAMHS policy. The group has published information about services, workforce, case-mix and more which is very helpful in strategic planning. This group is a potential source of engagement with the wider CAMHS Community. 


\section{Conclusions}

In relation to the terms of reference for this piece of work, it is difficult to separate out the out factors relating specifically to children under 16 from those in the older age group. At this stage it is probably more useful to consider both groups together as the legislation and codes of practice as currently set out apply equally to both.

4.1 The planned provision of 108 inpatient beds for a population of 4.45 million is generous by today's standards where the emphasis is in community care and care as close to the patient's home as possible. This compares to 48 planned beds for adolescents in Scotland and 9 child beds for a population of just over 5 million. England and Wales identified 900 beds for a population of 53.4 million in the NICAPS study of 2001 However it is not yet certain that all these planned beds in Ireland will be commissioned and the CAMHS Advisory group along with the Health Executive may wish to consult with stakeholders on the issue of how these beds should best be used in light of the relative poverty of community provision.

Ireland

Beds per million population
Scotland England and Wales

11.1 16.8

16.8 
Adult Services working together in locally negotiated arrangements.

4.3 The current inpatient units have begun working together in a networked fashion, sharing information about referrals, admission criteria, referral pathways etc. They see themselves very much as a national resource with each unit relating to their local catchment area more closely but admission to all units is available to all patients if required. It is less clear however that the community CAMHS teams share this view. This is potentially a positive way forward to provide equity of care across the country with standardization of protocols and referral and admission processes. It could provide clarity for community teams about the task of the units and reduce the waste of time currently experienced by teams through multiple referrals and negotiations. The inpatient units need to be responsive to the needs of the communities and involvement of community services in the inpatient unit network from time to time would be helpful.

4.4 It would be helpful if the inpatient units could adopt a similar network approach to with the community services within their regions. This would diminish the sense of being disconnected from the units and losing touch with patients while they are inpatients. It would also assist the inpatient units in prioritizing their clinical work and maintain up to date knowledge of what units and community teams can provide. Perhaps the use of technology, especially video conferencing would be beneficial here. (See example of a Scottish network laid out in appendix V).

4.5 It is difficult to see how the deadlines for admission of young people can be implemented without considerable development of community teams, especially intensive home treatment programmes or similar. Current staffing of community teams according to the most recent census of staff is at best half of what was recommended in Vision for Change. This clearly does not allow for a comprehensive out-patient CAMH Service up to $18^{\text {th }}$ birthday. Although this may be more achievable if all the 108 beds are in place, it is likely to be some years yet 
before this is the case. More Tier 4 service in the community may be preferred by some young people and obviate the necessity for admission altogether thus keeping young people in their own communities.

4.6 The situation in Limerick demonstrates what happens when emergency services are not backed up with either appropriate beds or intensive community support. Staff in Limerick have conscientiously attempted to put in place appropriate protocols for the admission of young people locally. Nevertheless, having visited the wards, particularly the adult ward, it is clear that these are not appropriate settings for the care of acutely ill adolescents. There would appear to be a low tolerance of risk and a high tolerance of admission in Limerick based on long standing practice and a perception of isolation. It is likely that considerable pressure from parents and other services leads to the admission of what in other places might be considered to be "social" admissions. The recent appointment of a consultant with specific responsibility for the older age group is already showing some signs of improving the situation and this may well improve further when the rest of her team takes up post. If this is borne out, then it is useful evidence of the efficacy of specialization within CAMHS teams. With regard to admissions to the paediatric ward, some of this practice seems to be no different to what is happening in other parts of the country except it is more transparent, as CAMHS Consultants are taking responsibility for the patients rather than paediatricians. There does however seem to be a proportion of patients admitted for what would be considered a CAMH inpatient period of assessment and treatment. This practice carries significant risks ( see 5.7 below) and is out of step with CAMH practice across the rest of the country.

4.7 Although there is a problem with rural communities and transport, there remains an issue with patients being reluctant to be admitted at a distance from their homes. There may be several reasons for this, not least that the stigma associated with mental illness makes it difficult for families to admit that there is a problem. It may also be difficult for clinicians to "sell" a unit at a distance particularly when they know that 
there may be a prolonged wait for a bed and families may be stressed by circumstances. Nevertheless, patients do seem to be willing to travel for other types of healthcare. This reflects a similar situation in Scotland where the balance between centralized and local services has long been debated. The three CAMH Inpatient Units in Scotland are all located close to the central belt with patients having to travel long distances to receive inpatient care; up to 150 miles by road and travel by plane or ferry in some cases. It would seem that local clinicians have a part to play in helping patients to understand why specialist care is worth travelling for and units also need to make a concerted effort to understand the challenges of the regions that they serve.

4.8 There would appear to be a lack of engagement of community care social work services and other partner agencies in offering support to children and families in crisis. This is a particular problem out of hours but even in hours it was hard to determine how services were joined up. With a lack of crisis solutions, it is inevitable that more of a burden will fall on CAMH Services to fill the gaps. Children and young people may therefore be receiving a mental health service inappropriately when their needs are in fact social. This unfairly labels young people and may potentially lead to stigmatization and discrimination.

\section{Recommendations}

1. It would be helpful for the Mental Health Commission to work with those delivering services to define operational criteria for "exceptional circumstances" for the admission of patients under 18 to adult centres. The timelines for reducing admissions of patients under the age of 18 years to adult units should not be changed.

2. Local services should be encouraged to develop clear and transparent protocols for any admission of children to adult wards. Such protocols should be negotiated between adult and CAMH Services and should be informed by views from users of services and their carers. 
3. Any policy that accepts the admission of children and young people to adult wards should clearly state how their educational, recreational and developmental needs are going to be met in an ageappropriate manner. Findings from the investigation suggest that despite clear attempts in implement this, adult wards are not in a position to appropriately meet the needs of this population.

4. The network of inpatient units should be encouraged to formalize their work and give consideration to becoming a managed care network or a more formal managed clinical network. This could perhaps be considered within the remit of the CAMHS Advisory group. Such formalization might include shared admission criteria, shared referral forms and discharge procedures; clear statements about equity of access and transparent criteria for prioritizing clinical need.

5. Regional groupings should develop closer working relationships with one another so that the inpatient units can be more responsive to local needs. This could perhaps be done using a framework similar to an obligate network (see Appendix V) or some such.

6. Consideration should be given as to whether it is appropriate for services to offer out of hours on call cover when there is no clear support from other agencies and no appropriate local beds available for admission. This clearly applies to the situation in Limerick but also pertains in other areas. It would be helpful to involve the newly appointed Executive Clinical directors in assessing the risks and benefits of out of hours provision within their areas.

7. The formal use of paediatric beds to care for children with mental illness is risky even with support from mental health services except for very short term care such as physical care of a severely ill anorexic patient. It is impossible to influence the ward milieu and the isolation inherent in being the only mental health patient in a ward is not conducive to good care. In addition, nursing staff seldom have mental health training and are unaccustomed to phenomenology in mental illness and the use of psychotropic medication. There are circumstances where admission to paediatric wards is appropriate such as physical care of patients whose health is compromised by their mental illness and very short term admission 
for assessment following self-harm. However it is not recommended that planned admissions to paediatric wards for the care and treatment of psychiatric illness is routine even under the consultant care of a child and adolescent psychiatrist.

8. The CAMHS Advisory group should take a lead role in helping CAMH Services to prioritize their workload with attention being given to developing balanced services that are, as far as possible, able to meet the needs of the most seriously ill young people as well as those with less severe disorder. This could be achieved by establishing a set of principles around which services could plan their development strategically. Issues such as agreed referral criteria for CAMHS, monitoring workforce and reviewing models of care could legitimately form part of this group's agenda. It will inevitably include a role as advocates for this group of patients. Hopefully the work of the advisory group will be one way to drive increase capacity in the workforce and continue the roll-out of the Vision for Change recommendations in challenging circumstances.

9. At a National level it would be helpful to engage all partner agencies (social work, education, primary care, voluntary sector, patients and their families) in developing strategic plans as to how children and young people with mental health needs at all levels of severity can be met. 


\section{Appendix I}

\section{Documents and other evidence considered.}

A Vision for Change; Report of the Expert Group on Mental Health Policy; The government of Ireland, 2006

Admission data from the Mental Health Commission

Annual report on Child and Adolescent Mental Health Services 2008;

CAMHS Advisory Group, Ireland.

Policies and procedures for the admission of a child to an Approved centre Limerick Mental Health Service August 2010-2012

Mental Health Act (Ireland) 2001

Code of Practice Relating to the Admission of Children; Mental Health Commission, 2006

Addendum to the Code of Practice Relating to the Admission of children, Mental Health Commission,2009

Various items of correspondence provided by the mental Health Commission

Health Advisory Services report “Together We Stand” (HMSO 1995)

Promotion, Prevention and Care; a Framework for Mental Health Services for Children and Young People; The Scottish Government, 2005

Needs Assessment of Tier 4 Adolescent Mental Health in the North of Scotland; NoSPHIN, 2010

NICAPS Study, Review of national Inpatient Child and Adolescent Psychiatry. O’Herlihy et al, The Royal College of Psychiatrists, 2001.

Limerick Mental Health Services, Policy documents an the care of children admitted to an Approved Centre, August 2010-2012 
Children and Young People in Mind, final report of the National CAMHS review, Department of Health 2008

Delivering for Remote and Rural Healthcare; The Final Report of the Remote and Rural Workstream, the Scottish Government, 2007. 


\section{Appendix II}

Figures on staffing levels, actual versus recommended

\begin{tabular}{|c|c|c|c|c|c|}
\hline $\begin{array}{l}\text { Vision for } \\
\text { Change } \\
\text { recommendation } \\
\text { (2006) }\end{array}$ & Recommended & Number & In Place & $\begin{array}{l}\text { Staff } \\
\text { recommended }\end{array}$ & $\begin{array}{l}\text { Staff in } \\
\text { Place }\end{array}$ \\
\hline $\begin{array}{l}\text { CAMHS } \\
\text { Community } \\
\text { teams }\end{array}$ & $1: 50,000$ & 84.7 & 49 & 1101.1 & 421.93 \\
\hline $\begin{array}{l}\text { Adolescent Day } \\
\text { hospitals }\end{array}$ & & (14) & 2 & & \\
\hline $\begin{array}{l}\text { Hospital Liaison } \\
\text { Teams }\end{array}$ & $1: 3000,000$ & 14.12 & 3 & 183.56 & $\begin{array}{l}\text { Not } \\
\text { available }\end{array}$ \\
\hline Inpatient beds & 108 & & $\begin{array}{l}52 \\
\text { (assuming } \\
\text { full } \\
\text { opening } \\
\text { of the } 2 \\
\text { new units } \\
\text { in Cork } \\
\text { and } \\
\text { Galway) }\end{array}$ & & \\
\hline
\end{tabular}




\section{Appendix III}

Figures on Admission of Children ( supplied by the Mental Health Commission)

\begin{tabular}{|c|c|c|c|c|}
\hline & $\begin{array}{l}\text { Number of } \\
\text { Admissions }\end{array}$ & $\begin{array}{l}\text { !5 years } \\
\text { and under }\end{array}$ & 16 years & 17 years \\
\hline $\begin{array}{l}\text { To } \\
\text { Adolescent } \\
\text { Units } \\
01 / 01 / 10- \\
30 / 06 / 10\end{array}$ & 134 & 64 & 43 & 27 \\
\hline $\begin{array}{l}\text { To Adult } \\
\text { Approved } \\
\text { Centres } \\
1 / 07 / 09- \\
31 / 12 / 09 \\
\end{array}$ & 94 & 1 & 26 & 67 \\
\hline $\begin{array}{l}\text { To Adult } \\
\text { Approved } \\
\text { Centres } \\
1 / 01 / 10- \\
30 / 06 / 10 \\
\end{array}$ & 91 & 11 & 28 & 52 \\
\hline $\begin{array}{l}\text { To } \\
\text { Adolescent } \\
\text { Units } 2008 \\
\end{array}$ & 143 & 93 & 31 & 19 \\
\hline $\begin{array}{l}\text { To Adult } \\
\text { Units } 2008\end{array}$ & 263 & 27 & 82 & 154 \\
\hline $\begin{array}{l}\text { To } \\
\text { Adolescent } \\
\text { Units } 2007\end{array}$ & 146 & 107 & 23 & 16 \\
\hline $\begin{array}{l}\text { To Adult } \\
\text { Units } 2007\end{array}$ & 218 & 14 & 70 & 134 \\
\hline $\begin{array}{l}\text { To } \\
\text { Adolescent } \\
\text { Units } 2006\end{array}$ & 145 & 112 & 20 & 13 \\
\hline $\begin{array}{l}\text { To Adult } \\
\text { Units } 2006\end{array}$ & 253 & 23 & 78 & 152 \\
\hline
\end{tabular}




\section{$\underline{\text { Appendix IV }}$}

\section{Names of those interviewed in compiling this report}

Dr. Patrick Devitt; Inspector of Mental Health Services

Dr. Brenden Doody; Mental Health Commission, Consultant Child and Adolescent Psychiatrist, HSE Lead for CAMHS

Mr. Martin Rogan; HSE Assistant National Director with responsibility for mental health services

(Both the above individuals have responsibilities that cover at least two organisations and were able to contribute to the information gathering from several perspectives).

Ms. Patricia Gilheaney, Director of Standards and Quality Assurance, Mental Health Commission

Dr. Keith Holmes, Consultant Child and Adolescent Psychiatrist, Chair of the Child and Adolescent Faculty of the College of Psychiatrists in Ireland

Mr. Hugh Kane; Chief Executive Mental Health Commission

Dr. Edmond O’Dea; Chair of Mental Health Commission

\section{Limerick CAMHS}

Dr. Rachel Davis

Dr. David Leahy

Dr. Susan O’Hanrahan

Dr. Eithne Foley

Phil Canny, Assisstant Director of Nursing

Catriona Hawley, Principal Social Worker

Teresa Bulfin, Manager

Nora Mullone, Charge Nurse, Ward 5B 


\section{Appendix V}

\section{An Example from one Scottish region for proposed delivery of Tier 4 Service in a rural area}

There is no nationally agreed definition of what constitutes Tier 4 services for CAMHS. Services are variously described as "Specialist”, "Inpatient”, "Daypatient", "Intensive community" and others. It has become clear as CAMH Services have developed and matured that not all young people with serious mental illness will require inpatient admission if adequate services are available in the community. For that reason, the North of Scotland has used a definition of need to define Tier 4 and uses the guide of "a young person who because of their mental illness requires the input of more than one clinician, more than once a week". Thus the definition relies on intensity of need rather than diagnosis or circumstances.

Over the past 3 years the North of Scotland Planning Group (NoSPG) has supported a project to re-provide the current inpatient unit based in Dundee with a new 12 bedded unit within the context of a community network. This is in line with the Scottish Government's commitment to provide 64 inpatient beds for Adolescent Psychiatry patients made in Delivering for mental Health. The proposed bed base is now smaller than this (48) as intensive Tier 4 Community services are also being developed. The NoSPG project envisages 12 inpatient beds in Dundee built in a flexible use model to enable the care of a variety of different patient groups. In addition to this, a network of clinicians working at Tier 4 level will be available to support patient before and after admission to the unit and, through accessing consultation and supervision, hopefully prevent the admission of some young people. The inpatient unit will act as a resource hub for the network which will be supported with the use of technology in the form of video conferencing, telephone conferencing and other IT applications for young people such as Facebook and MSN. The work of the Project has been informed by a needs assessment undertaken by the Public Health Department of NoSPG which recommended a mixed economy of Tier 4 support to include both inpatient and community resources.

There are 6 Health Boards in the North of Scotland Regional Group, 3 mainland boards and the 3 island boards of Orkney, Shetland and the Western Isles. In order to make the proposed provision work, an "Obligate 
Network” is proposed. This is an agreement between Health Boards around use and provision of services and as the name implies, places obligations on both sides to support an agreed service arrangement. In this case, there would be an obligation that the inpatient unit would provide access to beds in a transparent and equitable way to all 6 Boards in the network and there would be a reciprocal obligation on the Boards to provide adequate community resource to support young people through their admission and into discharge. The intention is that this would facilitate good through care and hopefully reduce length of inpatient stay. The tool proposed to make this work is an agreed Integrated Care Pathway that would lay out the expectations for standards of care at each stage in a patient's contact with services. The ICP would apply equally to inpatients and community Tier 4 patients and thus ensure that patients received the same quality of care regardless of their circumstances.

In order to provide good governance for the Inpatient Unit, it is expected that the Unit will remain an integral part of the National Inpatient Forum, a semi-formal network of the 3 Scottish inpatient units that meets regularly to agree policy and procedure across Scotland and to provide training and peer support for those working in adolescent inpatient settings. It should be notd that the single child inpatient unit in Scotland in a similar way is part of a managed clinical network for children with complex mental health need. 